\title{
Alternative Approaches to Study Mining and Mineral Science at ISOLDE-CERN
}

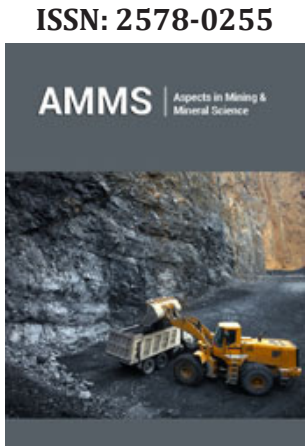

*Corresponding author: Schell J, European Organization for Nuclear Research (CERN), CH-1211 Geneva, Switzerland

Institute for Materials Science and Center for Nanointegration Duisburg-Essen (CENIDE), University of Duisburg-Essen, 45141 Essen, Germany

Submission: 侐 December 13, 2020

Published: 㱕March 11, 2020

Volume 4 - Issue 4

How to cite this article: Schell J. Alternative Approaches to Study Mining and Mineral Science at ISOLDECERN. Aspects Min Miner Sci.4(4). AMMS.000592.2020.

DOI: 10.31031/AMMS.2020.04.000592

Copyright@ Schell J, This article is distributed under the terms of the Creative Commons Attribution 4.0 International License, which permits unrestricted use and redistribution provided that the original author and source are credited.

\section{Schell J1,2*}

${ }^{1}$ European Organization for Nuclear Research, Switzerland

${ }^{2}$ Institute for Materials Science and Center for Nanointegration Duisburg-Essen (CENIDE), Germany

\begin{abstract}
ISOLDE-CERN is a large-scale facility for the on-line production of exotic radioactive isotopes, with high yields, high elemental selectivity and isotopic purity. Solid-state physics research at ISOLDE-CERN has been ongoing since 1976 and offers opportunities to conduct experiments using various material characterization techniques such as self-diffusion, photoluminescence, emission channelling, Mössbauer spectroscopy and perturbed angular correlations. The expertise of pioneers in our group and the variety of high-quality beams available at ISOLDE offer alternative approaches to research into mining and mineral science. This paper reviews the various beams, techniques and infrastructure that are available at ISOLDE-CERN and presents an investigation of diamond as a typical research example.
\end{abstract}

Keywords: ISOLDE-CERN; Perturbed angular correlations; Mössbauer spectroscopy; Self-diffusion; Photoluminescence; Emission channelling

Introduction

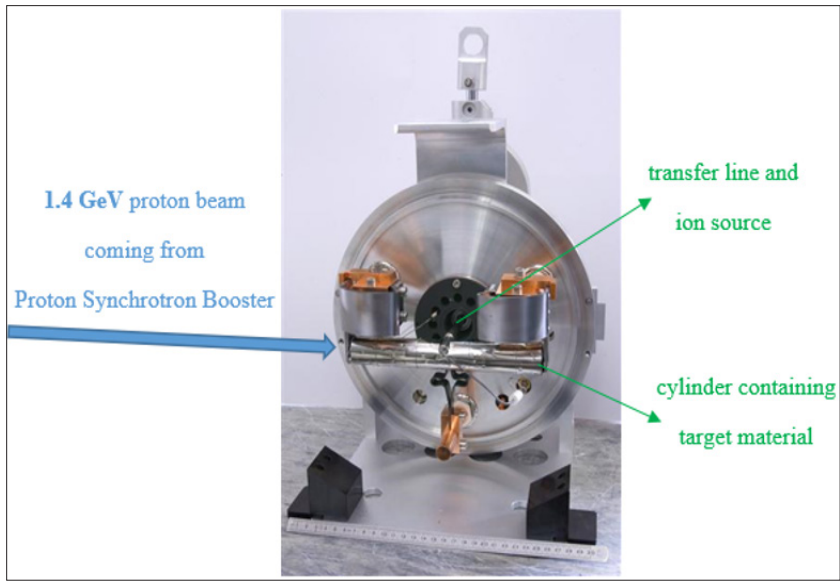

Figure 1: Typical target-ion-source unit used at ISOLDE-CERN. CERN is the copyright holder of the image (CERN-EX-0204023-01).

Mineral science involves the investigation of material properties including the chemical, crystal structure, physical and optical properties of minerals and mineralized artefacts. It is not feasible to create the important materials obtained by mining in a factory or laboratory. These materials are usually investigated by using conventional characterization techniques. However, ISOLDE-CERN offers more exotic techniques [1,2] that provide important additional information to help answer questions that cannot be answered by conventional methods. The solid-state physics programme at ISOLDE-CERN thus offers unique analytical instruments and expertise that complement traditional characterization techniques for optimizing materials for various applications. For example, to incorporate short-lived radioisotopes into materials, ISOLDE-CERN offers expertise and instrumentation for ion implantation and subsequent thermal annealing. The ISOLDE laboratory is a radioactive-ion-beam facility and is the oldest running installation at the CERN accelerator complex. The laboratory recently celebrated 50 years of successful operation with 40 years of production of high-quality beam for materials science research. The $1.4 \mathrm{GeV}$ proton beam from the Proton Synchrotron Booster at CERN is directed to independent target-ion-source units at ISOLDE (Figure 1). Depending on the requirements of specific experiments, ISOLDE can produce appropriate targets made 
of different materials and equipped with different types of ion sources. The ion sources used at ISOLDE are based on surface ionization, plasma ionization or a resonance ionization laser ion source. The target units are coupled to two conceptually different isotope separators. After mass separation, the beam is delivered to the beamlines at the ISOLDE hall. The equipment for materials science research is located in the low-energy-beam area, where the beam is accelerated up to $60 \mathrm{keV}$.

To illustrate the techniques available at ISOLDE-CERN, we now describe measurements performed in diamond. Implantation into diamond is an interesting subject motivated by the search for suitable n-type dopant atoms, which would lead to an ideal base for semiconducting devices for specific applications. Implanting dopant atoms into diamond allows us to tune several material properties, such as the high thermal conductivity, wide band gap, extreme hardness and high mobility for both p- and n-type carriers.

\section{Techniques}

Figure 2 shows photographs of some of the materialcharacterization setups available at ISOLDE-CERN. The optical and electronic properties of materials can be investigated by using the photoluminescence (PL) setup for materials implanted with radioactive isotopes [2,3]. Since ISOLDE-CERN can produce over 1000 different isotopes, the observation of either decay or growth related to implanted long- or short-lived radiotracers allows for distinct chemical identification of chemical species in several material types and compositions [4-6]. Recently, a study was proposed at ISOLDE to determine the route by which an unidentified phosphorus vacancy is produced via implantation of ${ }^{31} \mathrm{Al}$ in diamond [7]. The emission channelling (EC) technique provides information about the lattice location of isotopes implant in the single crystals $[8,9]$. ISOLDE-CERN is the only laboratory in the world that has an EC setup. The facility is the most convenient "home" for the EC technique, since it provides over 1000 different isotopes of most elements in the periodic table. For a given elemental dopant, the adequate radioactive isotope is selected and implanted into a single crystal. The EC measurements can be performed online or offline, depending on the half-life of the probe. Position- and energy-sensitive detectors are used to detect decay particles in the vicinity of major crystallographic directions and provide accurate information on the lattice location of the dopant. Depending on particle charge and on the lattice site of the radioactive dopants, decay particles such as $\alpha, \beta^{-}, \beta^{+}$and CE can be channelled or blocked on their route out of the crystal. The measurement can be performed as a function of thermal annealing, implantation fluency and temperature. The experimental patterns are determined by fitting via simulations for data interpretation. Even for different lattice sites, the lattice location of the dopant can be determined with unrivalled precision.

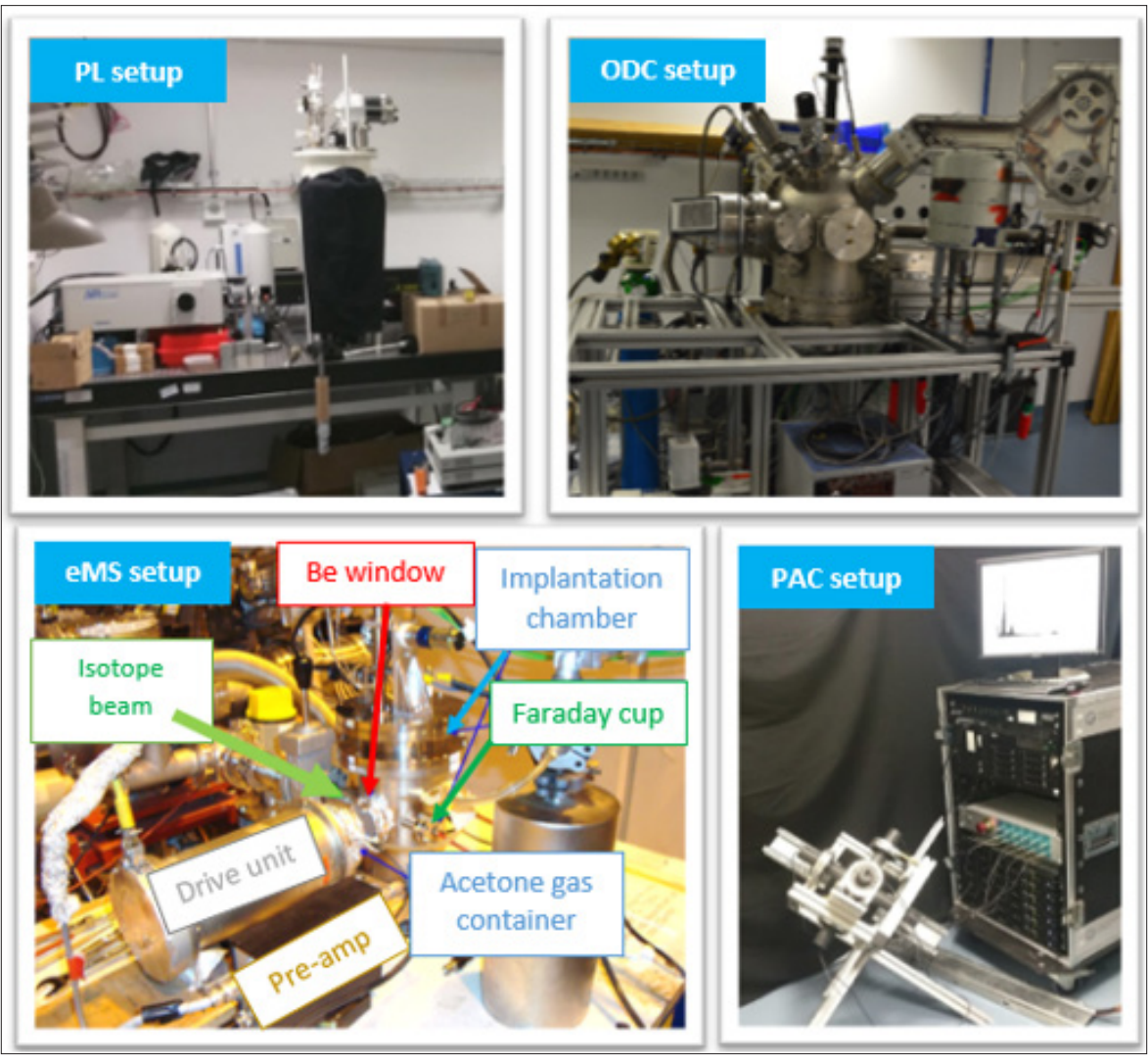

Figure 2: Four setups for material characterization that are currently used in the solid-state physics programme at ISOLDE-CERN. The photograph of the ODC chamber was taken by Mr Daniel Gaertner, University of Münster. 
Recently, the thermal stability of both interstitial and substitutional $\mathrm{Mn}$ in ferromagnetic $(\mathrm{Ga}, \mathrm{Mn}) \mathrm{As}$ was investigated by using the EC technique [9]. For instance, ${ }^{73} \mathrm{As}$-implanted diamond shows that thermal annealing above $1200 \mathrm{~K}$ leads to substitutional lattice occupation at a fraction of approximately 50\% [10,11]. The implantation was performed at energies of 120 and $60 \mathrm{keV}$ and with doses of $5 \times 10^{12}$ and $1 \times 10^{13}$ ions $/ \mathrm{cm}^{2}$ for ${ }^{111} \mathrm{In}$ and ${ }^{73} \mathrm{As}$, respectively [10]. The implantation of radioactive As ions was performed at room temperature at ISOLDE-CERN. About $40 \%$ of the In ions occupied substitutional lattice sites, whereas over $50 \%$ of As ions occupied these sites. Furthermore, the results obtained with the In probe show that implantation at $1373 \mathrm{~K}$ yields the same fraction of dopant ions at substitutional sites as does roomtemperature implantation [10]. The experiments performed using ${ }^{111}$ In and ${ }^{73} \mathrm{As}$ as probes show that the implantation damage induced by the heavy-ion implantation process is annealed in two distinct stages [10]. Mössbauer spectroscopy (MS) gives information on the electric-field gradient, magnetic hyperfine field, charge symmetry and binding properties $[12,13]$. ISOLDE-CERN, offers the possibility to carry out online experiments in which short-lived radioactive isotopes are produced, directly implanted into materials and measured at the beamline. Depending on the lifetime, the implantation and emission Mössbauer spectroscopy (eMS) measurements may occur at the same time or they may be separated in space and time. For example, the Mössbauer isotope ${ }^{119} \mathrm{Sn}$ presents a $24 \mathrm{keV}$ Mössbauer transition, which is populated in the decay of a ${ }^{119 m} \mathrm{Sn}$ isomeric state. At ISOLDE-CERN, the implantation of ${ }^{119} \mathrm{In}$ and ${ }^{119} \mathrm{Sb}$ probes as precursor isotopes into materials enables the controlled incorporation of ${ }^{119} \mathrm{Sn}$ probe atoms into a particular lattice site [12]. The MS technique was used at ISOLDE-CERN to study the incorporation, lattice location and annealing of defects of $\mathrm{Sb}$ and $\mathrm{Sn}$ ions in diamond [14]. The results obtained covered a broad distribution that reflected the implantation-induced lattice damage. The spectrum was fit with two symmetric doublet components. The samples were also measured as a function of annealing temperature and the analysis required a single line S1 with isomer shift $\delta=1.74(4) \mathrm{mm} / \mathrm{s}$ in addition to a weaker line at $0.52 \mathrm{~mm} / \mathrm{s}$. The increasing intensity of S1 evinced the recovery of lattice damage by thermal annealing. The results indicate that, after implantation at room temperature followed by thermal annealing above $1300 \mathrm{~K}, 40 \%$ of the ${ }^{119} \mathrm{Sn}$ implanted ion probes occupy or are close to regular lattice sites.

Perturbed angular correlations (PAC) experiments provide information about the local structure of matter via hyperfine interactions [15-17]. A conventional PAC experiment detects two $\gamma$ rays emitted in succession from a nuclear decay. The first is detected, selecting a spin-aligned ensemble; the second is then emitted from this ensemble anisotropically with respect to the first $\gamma$ ray. Here, because nuclear decay conserves angular momentum, the probability of a $\gamma$ ray being emitted in a certain direction depends on the orientation of the nuclear spin I. The spin orientations of the PAC probes initially are randomly distributed, so radiation is emitted isotropically in space. To observe an anisotropic angular distribution of radiation, an ensemble of nuclei is selected with an aligned spin distribution. This selection is done by detecting the first $\gamma$ ray of the cascade in a given direction. Note that the probability of emission of the second $\gamma$ ray is therefore anisotropic with respect to the direction of the first $\gamma$ ray, with an anisotropy coefficient that depends on the properties of the nuclear decay cascade. Once the PAC probe is incorporated into a crystal lattice, information about the local structure may be obtained via hyperfine interactions. Outside the ISOLDE-CERN laboratories, PAC measurements of diamond were performed using ${ }^{181} \mathrm{Hf}$ probes [18]. The samples consisted of (100) cleaved single-crystal diamond. Two distinct electric-field gradients, $\mathrm{V}_{\mathrm{zz}(1)}=5.5 \times 10^{21} \mathrm{~V} / \mathrm{m}^{2}$ and $\mathrm{V}_{\mathrm{zz}(2)}=9.6 \times 10^{21} \mathrm{~V} /$ $\mathrm{m}^{2}$, were observed in fractions of $5 \%$ for each local environment. The results show that these low probe fractions experience axially symmetric electric-field gradients. Moreover, the bulk of the probe ions is strongly disturbed by higher electric-field gradients. These results are consistent with measurements made using ${ }^{111}$ In probes [19]. The measurement made with the ${ }^{181} \mathrm{Hf}$ probes was crucial to complement the measurement with the ${ }^{111}$ In probes to verify the possible existence of after-effects $[20,21]$ coming from the electroncapture decay of ${ }^{111}$ In. Type-IIa diamond with a band gap of about $5.3 \mathrm{eV}$ is a perfect insulator and would likely allow such effects. Later, the measurement results were compared with experimental results obtained using ${ }^{111 \mathrm{~m}} \mathrm{Cd}$ probes [22]. The results indicate that the loss of anisotropy in the PAC signal is not due to after-effects but to extended lattice damage produced by the implantation process that remained even after thermal annealing at high temperatures. Therefore, the authors suggest that low-dose implantation at a range of energies is more appropriate for dopant incorporation at regular, defect-free lattice sites.

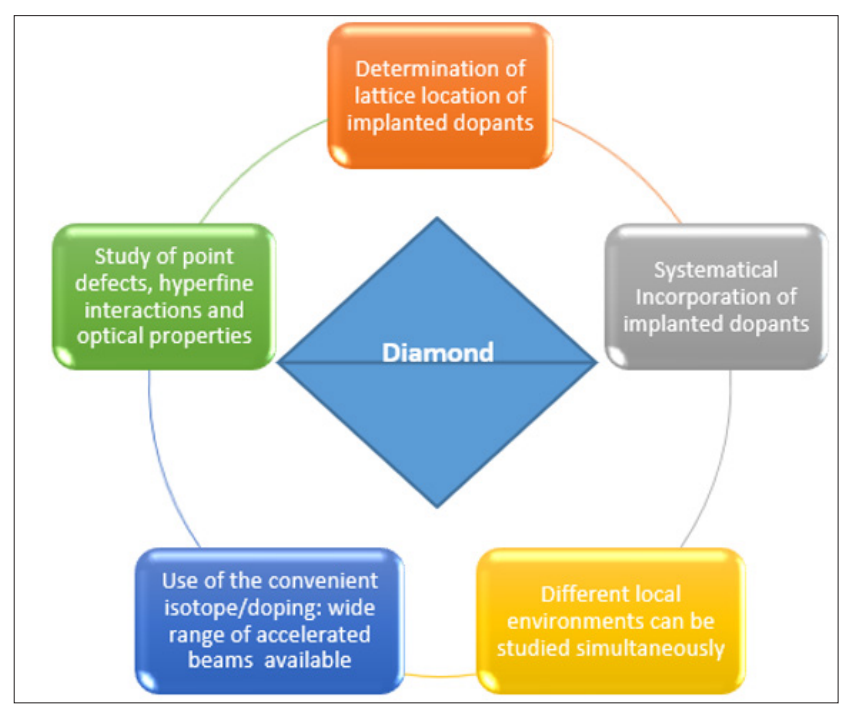

Figure 3: Smart graphic indicating the scientific goals that can be achieved by using the characterization techniques available at ISOLDECERN for materials science (not only for diamond).

Radiotracer diffusion provides information about the thermal motion of lattice ions and is the oldest technique used in nuclear solid-state physics [23]. At ISOLDE-CERN, the Online Diffusion Chamber (ODC) serves to measure the diffusion profile of 
radioactive isotopes in materials, even if probes are short lived. The study of how host elements self-diffuse in a crystalline lattice is of considerable importance for materials science (Figure 3). The ODC allows for a very direct measurement of diffusion profiles, which is highly indicative of the diffusion mechanism in the material of interest. The self-diffusion method [24] has yet to be used for investigating diamond. Recently, the technique was used to study the incorporation of $\mathrm{Cd}$ into a titanium dioxide lattice [25]. For instance, this radiotracer technique was used to investigate the diffusion of all constituent elements in equi-atomic CoCrFeNi and CoCrFeMnNi [24]. The constituent elements have significantly different diffusion rates, with $\mathrm{Mn}$ diffusing the fastest and $\mathrm{Ni}$ and Co the slowest.

\section{Conclusion}

This short communication describes various possible applications of radioactive-ion beams produced at the ISOLDECERN facility in nuclear solid-state physics and for studying the incorporation of probe atoms into solids. Having been successfully applied in diamond, these methodscan also be used for investigations in mining and mineral science. The incorporation of radioactive isotopes can be controlled systematically by implantation and annealing. A careful annealing procedure gradually corrects defect bonds between carbon atoms, thereby leading to the recovery of the regular lattice structure in the local environment of the probe isotopes. To propose investigations of materials at ISOLDE-CERN, please contact the physics coordinator and the solid-state physics coordinator [26]. Scientific proposals are evaluated by the ISOLDE and Neutron Time-of-Flight Committee (INTC) [27].

\section{Acknowledgement}

This research was funded by the Federal Ministry of Education and Research (BMBF) through Grant 05K16PGA.

\section{References}

1. Catherall R, Andreazza W, Breitenfeldt M, Dorsival A, Focker GJ, et al (2017) The ISOLDE facility. Journal of Physics G 44(9): 094002-094020.

2. Johnston K, Schell J, Correia JG, Deicher M, Gunnlaugsson HP, et al. (2017) The solid state physics programme at ISOLDE: recent developments and perspectives. Journal of Physics G 44(10): 104001-104026.

3. Broser I, Franke KH (1965) Journal of Physical Chemistry of Solids 26: 1013.

4. Johnston K, Henry MO, Mc Cabe D, Dietrich M, Alves E (2006) Physical Review B 73: 165212.

5. Johnston K, Cullen J, Henry M, McGlynn E, Stachura M (2011) Evidence for As lattice location and Ge bound exciton luminescence in $\mathrm{ZnO}$ implanted with ${ }^{73} \mathrm{As}$ and ${ }^{73} \mathrm{Ge}$. Physical Review B 83(12): 125205.

6. Cullen J, Byrne D, Johnston K, McGlynn E, Henry MO (2013) Chemical identification of luminescence due to $\mathrm{Sn}$ and $\mathrm{Sb}$ in $\mathrm{ZnO}$. Applied Physics Letters 102(19): 192110.

7. Green BL, Newton ME, Johnston K (2018) Production of phosphorus vacancy centers in diamond for optical and spin characterization. Proposal to the ISOLDE and Neutron Time-of-Flight Committee, pp. 1-9.
8. Hofsäss H, Lindner G (1991) Physics Reports 20: 123.

9. Lima TAL, Wahl U, Costa A, Augustyns V, Edmonds KW, et al. (2019) Thermal stability of interstitial and substitutional Mn in ferromagnetic (Ga,Mn)As. Physical Review B 100(14): 144409.

10. Quintel H, Bharuth Ram K, Hofsäss H, Restle M, Ronning C (1996) Emission channeling study of annealing of radiation damage in heavyion implanted diamond. Nuclear Instrumentation Methods B 118(1-4): 72-75.

11. Correia JG, Marques JG, Alves E, Forkel Wirth D, Jahn SG, et al. (1997) Microscopic studies of implanted ${ }^{73}$ As in diamond. Nuclear Instrumentation Methods B 127-128: 723-726.

12. Weyer G and the ISOLDE Collaboration (2000) Mössbauer spectroscopy at ISOLDE. Hyperfine Interactions 129: 371-390.

13. Weyer G (2007) Defects in semiconductors- results from Mössbauer spectroscopy. Hyperfine Interactions 177: 1-13.

14. Bharuth Ram K, Gunnlaugsson HP, Masenda H, Sielemann R, Weyer G, et al. (2012) Defect annealing in Sb/Sn implanted diamond investigated with ${ }^{119} \mathrm{Sn}$ Mössbauer spectroscopy. Physica B: Physics of Condensed Matter 407(15): 2923-2925.

15. Schell J, Schaaf P, Lupascu DC (2017) Perturbed angular correlations at ISOLDE: A 40 years young technique. AIP Advances 7(10): 105017.

16. Abragam A, Pound RV (1953) Influence of electric and magnetic fields on angular correlations. Physical Review 92(4): 943.

17. Frauenfelder H, Steffen RM, Siegbahn K (1965) $\alpha$-, $\beta$ - and $\gamma$-ray spectroscopy, In: Siegbahn K (Ed.), North-Holland, Netherlands.

18. Raudies JH, Appel H, Then GM, Thies WG, Freitag K, et al. (1983) TDPAC studies on ${ }^{181} \mathrm{Hf}$ implanted into diamond. Hyperfine Interactions 15: 487-490.

19. Appel H, Raudies J, Thies WG, Hanser A, Sellschop JPF (1981) Residence sites for ${ }^{111} \mathrm{ln}$ implanted in diamond. Hyperfine Interactions 10: 735.

20. Lupascu D, Habenicht S, Lieb KP, Neubauer M, Uhrmacher M, et al. (1996) Relaxation of electronic defects in pure and doped La203 observed by perturbed angular correlations. Phys Rev B Condens Matter 54(2): 871883.

21. Dang TT, Schell J, Lupascu DC, Vianden R (2018) Dynamic quadrupole interactions in semiconductors. Journal of Applied Physics 123(16): 165109.

22. Bharuth Ram K, Burchard A, Deicher M, Quintel H, Restle M, et al. (2001) Implantation sites of In, Cd, and Hf ions in diamond. Physical Review B 64(19): 195207.

23. Groh J, Hevesey GV (1920) Annals of Physics 65: 218.

24. Gaertner D, Kottke J, Wilde G, Divinski SV, Chumlyakov Y (2018) Tracer diffusion in single crystalline CoCrFeNi and CoCrFeMnNi high entropy alloys. Journal of Materials Research 33(19): 3184-3191.

25.Zyabkin DV, Schell J, Gaertner D, Dang TT, Gonçalves JN, et al. (2019) Hyperfine interactions and diffusion of $\mathrm{Cd}$ in $\mathrm{Tio}_{2}$ (rutile). Journal of Applied Physics 126(1): 015102.

26. The ISOLDE Radioactive Ion Beam facility. Contact for information available at https://isolde.web.cern.ch/

27. ISOLDE and Neutron Time-of-Flight Experiments Committee. http:// committees.web.cern.ch/intc/

For possible submissions Click below: 